\title{
The zeros of random polynomials cluster uniformly near the unit circle
}

\author{
C. P. Hughes and A. Nikeghbali
}

\begin{abstract}
In this paper we deduce a universal result about the asymptotic distribution of roots of random polynomials, which can be seen as a complement to an old and famous result of Erdős and Turan. More precisely, given a sequence of random polynomials, we show that, under some very general conditions, the roots tend to cluster near the unit circle, and their angles are uniformly distributed. The method we use is deterministic: in particular, we do not assume independence or equidistribution of the coefficients of the polynomial.
\end{abstract}

\section{Introduction}

In this paper, we are interested in the uniform concentration near the unit circle of roots of polynomials.

Let $\left(P_{N}(Z)\right)_{N \geqslant 1}$ be a sequence of polynomials. Denote the zeros of $P_{N}(Z)$ by $z_{1}, \ldots, z_{N}$. Let

$$
\nu_{N}(\rho):=\#\left\{z_{k}: 1-\rho \leqslant\left|z_{k}\right| \leqslant \frac{1}{1-\rho}\right\}
$$

be the number of zeros of $P_{N}(Z)$ lying in the annulus bounded by $1-\rho$ and $1 /(1-\rho)$, where $0 \leqslant \rho \leqslant 1$, and let

$$
\nu_{N}(\theta, \phi):=\#\left\{z_{k}: \theta \leqslant \arg \left(z_{k}\right)<\phi\right\}
$$

be the number of zeros of $P_{N}(Z)$ whose argument lies between $\theta$ and $\phi$, where $0 \leqslant \theta<\phi \leqslant 2 \pi$.

We say that the zeros cluster uniformly around the unit circle if for all fixed $0<\rho<1$,

$$
\lim _{N \rightarrow \infty} \frac{1}{N} \nu_{N}(\rho)=1
$$

and

$$
\lim _{N \rightarrow \infty} \frac{1}{N} \nu_{N}(\theta, \phi)=\frac{\phi-\theta}{2 \pi} .
$$

The purpose of this paper is to find a general but simple condition for when the zeros cluster uniformly around the unit circle.

Theorem 1. Let $\left(P_{N}(Z)\right)$ be a sequence of polynomials, with

$$
P_{N}(Z)=\sum_{k=0}^{N} a_{N, k} Z^{k},
$$

Received 3 July 2006, accepted in final form 24 August 2007, published online 14 March 2008. 2000 Mathematics Subject Classification 30C15.

Keywords: random polynomials, roots, uniform clustering.

This journal is (C) Foundation Compositio Mathematica 2008. 


\section{ZEROS OF RANDOM POLYNOMIALS}

such that $a_{N, 0} a_{N, N} \neq 0$ for all $N$. Let

$$
L_{N}\left(P_{N}\right)=\log \left(\sum_{k=0}^{N}\left|a_{N, k}\right|\right)-\frac{1}{2} \log \left|a_{N, 0}\right|-\frac{1}{2} \log \left|a_{N, N}\right| .
$$

If

$$
L_{N}\left(P_{N}\right)=o(N)
$$

then the zeros of this sequence cluster uniformly near the unit circle, i.e. for all $0<\rho<1$,

$$
\lim _{N \rightarrow \infty} \frac{1}{N} \nu_{N}(\rho)=1
$$

and for all $0 \leqslant \theta<\phi \leqslant 2 \pi$,

$$
\lim _{N \rightarrow \infty} \frac{1}{N} \nu_{N}(\theta, \phi)=\frac{\phi-\theta}{2 \pi} .
$$

where $\nu_{N}(\rho)$ and $\nu_{N}(\theta, \phi)$ are defined in (1) and (2) respectively.

The second part of our theorem, on $\nu_{N}(\theta, \phi)$, follows from the celebrated result of Erdős and Turan [ET50] on the distribution of roots of polynomials.

Theorem 2 (Erdős-Turan). Let $\left(a_{k}\right)_{0 \leqslant k \leqslant N}$ be a sequence of complex numbers such that $a_{0} a_{N} \neq 0$, and let

$$
P(Z)=\sum_{k=0}^{N} a_{k} Z^{k}
$$

Define

$$
L_{N}(P)=\log \sum_{k=0}^{N}\left|a_{k}\right|-\frac{1}{2} \log \left|a_{0}\right|-\frac{1}{2} \log \left|a_{N}\right| .
$$

Then

$$
\left|\frac{1}{N} \nu_{N}(\theta, \phi)-\frac{\phi-\theta}{2 \pi}\right|^{2} \leqslant \frac{C}{N} L_{N}(P)
$$

for some constant $C$, where $\nu_{N}(\theta, \phi)$ is defined in (2).

The above theorem shows that if $L_{N}(P)$ is small compared with the degree $N$, then the angles of the roots are nearly uniformly distributed, and that is precisely the reason why this theorem has been extensively used to prove asymptotic uniform concentration near the unit circle of the roots of some families of random polynomials.

In this paper, we prove a natural complement to this result.

Theorem 3. Let $\left(a_{k}\right)_{0 \leqslant k \leqslant N}$ be a sequence of complex numbers such that $a_{0} a_{N} \neq 0$, and let

$$
P(Z)=\sum_{k=0}^{N} a_{k} Z^{k}
$$

Let $L_{N}(P)$ be defined as in (5). Then for $0<\rho<1$,

$$
\left(1-\frac{1}{N} \nu_{N}(\rho)\right) \leqslant \frac{2}{N \rho} L_{N}(P)
$$

where $\nu_{N}(\rho)$ is defined in (1).

These two theorems on deterministic polynomials give a sufficient condition for the roots of random polynomials to cluster uniformly around the unit circle, and we show how results of Šparo 


\section{P. Hughes and A. Nikeghbali}

and Šur [SS62], Arnold [Arn66], and Shmerling and Hochberg [SH02] follow as corollaries of this. Indeed, we show that some of their conditions on the coefficients of the polynomials can be dropped.

More precisely, let $(\Omega, \mathcal{F}, \mathbb{P})$ be a probability space, on which a random array $\left(a_{N, k}\right)_{0 \leqslant k \leqslant N}$ is defined. Consider now the sequence of random polynomials $\left(P_{N}(Z)\right)$, with

$$
P_{N}(Z)=\sum_{k=0}^{N} a_{N, k} Z^{k} .
$$

The asymptotics for roots of random polynomials in the complex plane have already been studied in the special case where

$$
P_{N}(Z)=\sum_{k=0}^{N} a_{k} Z^{k} ;
$$

which corresponds to the special array $\left(a_{N, k}\right)_{k \leqslant N}=\left(a_{0}, a_{1}, \ldots, a_{N}\right)$, and it is not our intention to give a full historical account here (see [BS86], [Far98], [EK95] for more details and references), but we rather mention the papers of Šparo and Sur [SS62], Arnold [Arn66], and Shmerling and Hochberg [SH02] which contain the most general results about uniform clustering near the unit circle. Šparo and Šur [SS62] have considered i.i.d. complex coefficients $\left(a_{n}\right)_{n \geqslant 0}$ and have shown that under some integrability conditions, the zeros of such sequences cluster uniformly near the unit circle, with convergence in (3) and (4) holding in probability. Arnold [Arn66] improved this result and proved that the convergence holds almost surely and in the $p$ th mean if the moduli of $a_{k}$ are equidistributed (plus some integrability conditions). Recently, Shmerling and Hochberg [SH02] have obtained stronger results: they have shown that the condition on equidistribution can be dropped if $\left(a_{n}\right)_{n \geqslant 0}$ is a sequence of independent variables which have continuous densities $f_{n}$ which are uniformly bounded in some neighbourhood of the origin with finite means $\mu_{n}$ and standard deviations $\sigma_{n}$ that satisfy the condition

$$
\begin{gathered}
\max \left\{\limsup _{n \rightarrow \infty} \sqrt[n]{\left|\mu_{n}\right|}, \limsup _{n \rightarrow \infty} \sqrt[n]{\left|\sigma_{n}\right|}\right\}=1, \\
\mathbb{P}\left\{a_{0}=0\right\}=0
\end{gathered}
$$

The authors mentioned above consider the cases of (3) and (4) separately. They prove (4) using Theorem 2 and prove (3) using techniques from random power series. In particular, to prove (4), they are able to show that $L_{N}(P) / N \rightarrow 0$ as $N \rightarrow \infty$ and this proof is only a few lines, while the techniques used to prove (3) are more sophisticated. Thus, a side benefit of Theorem 3 is that once one proves the uniform distribution of the angles using Theorem 2 of Erdös and Turan, then one actually proves uniform clustering near the unit circle considerably simplifying the arguments in the proofs on uniform clustering in [Arn66] and [SH02]. Moreover, as we shall see, our results also give us an estimate for the rate of clustering (that is, how quickly $\rho \rightarrow 0$ as a function of $N)$.

The historical results we have mentioned above do not apply to the more general case of sequences of random polynomials of the form (6) we are dealing with, and they also do not lead to anything interesting in the case of deterministic sequences of polynomials (the asymptotic study of roots sequences of deterministic polynomials occurs in problems of equidistribution of algebraic integers; see, for example, [Bil97]). Moreover, these results do not cover the cases where the coefficients are dependent with different distributions: for example, in the semiclassical approximations for multidimensional quantum systems, one needs to locate roots of high-degree random polynomials with dependent and non-identically distributed coefficients [BBL96] (we should point out that the authors in [BBL96] have observed the uniform clustering in the special case of self-reciprocal polynomials with complex Gaussian coefficients with finite variance). 


\section{ZEROS OF RANDOM POLYNOMIALS}

The aim of this paper is to show that the phenomenon of uniform concentration of zeros around the unit circle is universal, in the sense that no independence or equidistribution on the coefficients is required, but only conditions on their size. Our method, based on elementary complex analysis, reduces both convergences (3) and (4) to the same problem, namely showing that $L_{N}(P)$, defined in (5), is small compared with the degree $N$ of the polynomial $P$, thus complementing Theorem 2 of Erdős and Turan.

The structure of this paper is as follows. In $\S 2$ we prove our main theorems and then, in $\S 3$, we use them to deduce clustering of zeros for general sequences of random polynomials.

\section{Basic estimates}

For $N \geqslant 1$, let $\left(a_{k}\right)_{0 \leqslant k \leqslant N}$ be a sequence of complex numbers satisfying $a_{0} a_{N} \neq 0$. From this sequence construct the polynomial

$$
P_{N}(Z)=\sum_{k=0}^{N} a_{k} Z^{k}
$$

and denote its zeros by $z_{i}$ (where $i$ ranges from 1 to $N$ ). For $0 \leqslant \rho \leqslant 1$, we are interested in estimates for

$$
\begin{gathered}
\widetilde{\nu}_{N}(\rho)=\#\left\{z_{j},\left|z_{j}\right|<1-\rho\right\}, \\
\bar{\nu}_{N}(\rho)=\#\left\{z_{j},\left|z_{j}\right|>\frac{1}{1-\rho}\right\}, \\
\nu_{N}(\rho)=\#\left\{z_{j}, 1-\rho \leqslant\left|z_{j}\right| \leqslant \frac{1}{1-\rho}\right\},
\end{gathered}
$$

which counts the number of zeros of the polynomial $P_{N}(Z)$ which lie respectively inside the open disc of radius $1-\rho$, outside the closed disc of radius $1 /(1-\rho)$, and inside the closed annulus bounded by circles of radius $1-\rho$ and $1 /(1-\rho)$.

Theorem 4 . For $N \geqslant 1$, let $\left(a_{k}\right)_{0 \leqslant k \leqslant N}$ be a sequence of complex numbers which satisfy $a_{0} a_{N} \neq 0$. Then, for $0<\rho<1$

$$
\begin{aligned}
& \frac{1}{N} \widetilde{\nu}_{N}(\rho) \leqslant \frac{1}{N \rho}\left(\log \left(\sum_{k=0}^{N}\left|a_{k}\right|\right)-\log \left|a_{0}\right|\right) \\
& \frac{1}{N} \bar{\nu}_{N}(\rho) \leqslant \frac{1}{N \rho}\left(\log \left(\sum_{k=0}^{n}\left|a_{k}\right|\right)-\log \left|a_{N}\right|\right)
\end{aligned}
$$

and

$$
\left(1-\frac{1}{N} \nu_{N}(\rho)\right) \leqslant \frac{2}{N \rho}\left(\log \left(\sum_{k=0}^{N}\left|a_{k}\right|\right)-\frac{1}{2} \log \left|a_{0}\right|-\frac{1}{2} \log \left|a_{N}\right|\right)
$$

Proof. An application of Jensen's formula (see, for example, [Lan99, p. 341]) yields

$$
\frac{1}{2 \pi} \int_{0}^{2 \pi} \log \left|P_{N}\left(e^{\mathrm{i} \varphi}\right)\right| \mathrm{d} \varphi-\log \left|P_{N}(0)\right|=\sum_{\left|z_{i}\right|<1} \log \frac{1}{\left|z_{i}\right|}
$$

where the sum on the right-hand side is on zeros lying inside the open unit disk. We have the 


\section{P. Hughes and A. Nikeghbali}

following minorization for this sum:

$$
\begin{aligned}
\sum_{\left|z_{i}\right|<1} \log \frac{1}{\left|z_{i}\right|} & \geqslant \sum_{\left|z_{i}\right|<1-\rho} \log \frac{1}{\left|z_{i}\right|} \\
& \geqslant \rho \widetilde{\nu}_{N}(\rho)
\end{aligned}
$$

since if $0 \leqslant \rho \leqslant 1$, then for all $\left|z_{i}\right| \leqslant 1-\rho, \log \left(1 /\left|z_{i}\right|\right) \geqslant \rho$, and by definition there are $\widetilde{\nu}_{N}(\rho)$ such terms in the sum.

We also have the following trivial upper bound

$$
\max _{\varphi \in[0,2 \pi]}\left|P_{N}\left(e^{\mathrm{i} \varphi}\right)\right| \leqslant \sum_{k=0}^{N}\left|a_{k}\right|,
$$

and so

$$
\begin{aligned}
\rho \widetilde{\nu}_{N}(\rho) & \leqslant \frac{1}{2 \pi} \int_{0}^{2 \pi} \log \left|P_{N}\left(e^{\mathrm{i} \varphi}\right)\right| \mathrm{d} \varphi-\log \left|a_{0}\right| \\
& \leqslant \log \left(\sum_{k=0}^{N}\left|a_{k}\right|\right)-\log \left|a_{0}\right|
\end{aligned}
$$

which gives (7).

To estimate the number of zeros lying outside the closed disc of radius $(1-\rho)^{-1}$, note that if $z_{0}$ is a zero of the polynomial $P_{N}(Z)=\sum_{k=0}^{N} a_{k} Z^{k}$, then $1 / z_{0}$ is a zero of the polynomial $Q_{N}(Z):=Z^{N} P_{N}(1 / Z)=a_{N}+a_{N-1} Z+\cdots+a_{0} Z^{N}$. Therefore, the number of zeros of $P_{N}(Z)$ outside the closed disc of radius $1 /(1-\rho)$ equals the number of zeros of $Q_{N}(Z)$ inside the open disc of radius $1-\rho$. Therefore, from (7) we obtain

$$
\frac{1}{N} \bar{\nu}_{N}(\rho) \leqslant \frac{1}{N \rho}\left(\log \left(\sum_{k=0}^{N}\left|a_{k}\right|\right)-\log \left|a_{N}\right|\right)
$$

which gives (8).

Since

$$
N-\nu_{N}(\rho)=\widetilde{\nu}_{N}(\rho)+\bar{\nu}_{N}(\rho)
$$

we immediately obtain (9).

Theorem 3 is merely a restatement of (9). Theorem 1 follows from combining this with the result of Erdős and Turan, Theorem 2.

Remark. Note that if $a_{k} \mapsto \lambda a_{k}$ for some $\lambda \neq 0$, then the zeros of $P_{N}(Z)$ are unchanged, and

$$
\log \left(\sum_{k=0}^{N}\left|\lambda a_{k}\right|\right)-\frac{1}{2} \log \left|\lambda a_{0}\right|-\frac{1}{2} \log \left|\lambda a_{N}\right|=\log \left(\sum_{k=0}^{N}\left|a_{k}\right|\right)-\frac{1}{2} \log \left|a_{0}\right|-\frac{1}{2} \log \left|a_{N}\right|
$$

so, in some sense, this is a natural function to control the location of the zeros.

\section{Uniform clustering results for roots of random polynomials}

We require no independence restriction on our random variables. We only assume that

$$
\mathbb{P}\left\{a_{N, 0}=0\right\}=0
$$

and

for all $N$.

$$
\mathbb{P}\left\{a_{N, N}=0\right\}=0,
$$




\section{ZEROS OF RANDOM POLYNOMIALS}

\subsection{The main theorem for random polynomials}

Theorem 5. For $N \geqslant 1$, let $\left(a_{N, k}\right)_{0 \leqslant k \leqslant N}$ be an array of random complex numbers such that $\mathbb{P}\left\{a_{N, 0}=0\right\}=0$ and $\mathbb{P}\left\{a_{N, N}=0\right\}=0$ for all $N$. Let

$$
L_{N}=\log \left(\sum_{k=0}^{N}\left|a_{N, k}\right|\right)-\frac{1}{2} \log \left|a_{N, 0}\right|-\frac{1}{2} \log \left|a_{N, N}\right| .
$$

If

$$
\mathbb{E}\left[L_{N}\right]=o(N) \quad \text { as } N \rightarrow \infty,
$$

then there exists a positive function $\alpha_{N}$ satisfying $\alpha_{N}=o(N)$ such that the zeros of the random polynomial

$$
P_{N}(Z)=\sum_{k=0}^{N} a_{N, k} Z^{k}
$$

satisfy

$$
\lim _{N \rightarrow \infty} \mathbb{E}\left[\frac{1}{N} \nu_{N}\left(\frac{\alpha_{N}}{N}\right)\right]=1
$$

and

$$
\lim _{N \rightarrow \infty} \mathbb{E}\left[\frac{1}{N} \nu_{N}(\theta, \phi)\right]=\frac{\phi-\theta}{2 \pi} .
$$

In fact the convergence also holds in probability and in the pth mean, for all positive $p$.

Furthermore, if there exists a (deterministic) positive function $\alpha_{N}$ satisfying $\alpha_{N} \leqslant N$ for all $N$, such that

$$
L_{N}=o\left(\alpha_{N}\right) \text { almost surely, }
$$

then both convergences hold almost surely (and also in the pth mean, for all positive $p$ ).

Proof. The convergence in mean for $\nu_{N}\left(\alpha_{N} / N\right)$ is a consequence of (9). We have

$$
1-\mathbb{E}\left[\frac{1}{N} \nu_{N}\left(\frac{\alpha_{N}}{N}\right)\right] \leqslant \frac{2}{\alpha_{N}} \mathbb{E}\left[L_{N}\right] .
$$

Therefore, we see that the result follows for any positive function $\alpha_{N}$ satisfying $\alpha_{N} \leqslant N$ for all $N$ such that $\mathbb{E}\left[L_{N}\right] / \alpha_{N} \rightarrow 0$, and such a function exists by assumption (13), for example

$$
\alpha_{N}=N \min \left\{1, \sqrt{\frac{\mathbb{E}\left[L_{N}\right]}{N}}\right\} .
$$

Similarly from Theorem 2 and (13) we have that

$$
\begin{aligned}
\mathbb{E}\left[\left|\frac{1}{N} \nu_{N}(\theta, \phi)-\frac{\phi-\theta}{2 \pi}\right|^{2}\right] & \leqslant \frac{C}{N} \mathbb{E}\left[L_{N}\right] \\
& =o(1) .
\end{aligned}
$$

Note that the mean square convergence implies convergence in the mean, as in the theorem, and also convergence in probability. Note, further, that since the random variables are uniformly bounded $\left(0 \leqslant(1 / N) \nu_{N}(\theta, \phi) \leqslant 1\right)$, mean convergence implies convergence in the $p$ th mean for all positive $p$.

In the same way, the almost sure convergence of $(1 / N) \nu_{N}\left(\alpha_{N} / N\right)$ and $(1 / N) \nu_{N}(\theta, \phi)$ follows immediately from (9) and Theorem 2, using (14). 


\section{P. Hughes and A. Nikeghbali}

In the following sections, we give some sufficient conditions, which are easy to check, for Theorem 5 to hold. We first consider the case of general sequences of random polynomials for which there exist no previous results to our knowledge; then we deal with the classical random polynomials.

\subsection{General sequences of random polynomials}

Proposition 6. Let $\left(a_{N, k}\right)$ be an array of random complex numbers which satisfy (10) and (11). Assume that $\mathbb{E}\left[\log \left|a_{N, 0}\right|\right]=o(N)$, and $\mathbb{E}\left[\log \left|a_{N, N}\right|\right]=o(N)$, and that there exists a fixed $0<s \leqslant 1$ such that

$$
\sum_{k=0}^{N} \mathbb{E}\left[\left|a_{N, k}\right|^{s}\right]=\exp (o(N)) .
$$

Then there exists a positive sequence $\left(\alpha_{N}\right)$ subject to $\alpha_{N}=o(N)$ such that

$$
\lim _{N \rightarrow \infty} \mathbb{E}\left[\frac{1}{N} \nu_{N}\left(\frac{\alpha_{N}}{N}\right)\right]=1
$$

and

$$
\lim _{N \rightarrow \infty} \mathbb{E}\left[\left|\frac{1}{N} \nu_{N}(\theta, \phi)-\frac{\phi-\theta}{2 \pi}\right|\right]=0 .
$$

Proof. Since $0<s \leqslant 1$ we have the following concavity inequality:

$$
\mathbb{E}\left[\log \left(\sum_{k=0}^{N}\left|a_{N, k}\right|\right)\right] \leqslant \frac{1}{s} \log \left(\sum_{k=0}^{N} \mathbb{E}\left[\left|a_{N, k}\right|^{s}\right]\right) .
$$

By assumption, the right-hand side is $o(N)$. Therefore $L_{N}$, defined in (12), satisfies $L_{N}=o(N)$, and the result follows from Theorem 5 .

Remark. The proposition shows that under some very general conditions (just some conditions on the size of the expected values of the modulus of the coefficients), without assuming any independence or equidistribution condition, the zeros of random polynomials tend to cluster uniformly near the unit circle. We can also remark that we do not assume that our coefficients must have density functions: they can be discrete-valued random variables.

Now we give two examples which could not be dealt with using the previous results available in the literature.

Example 1. Let $a_{N, k}$ be random variables distributed according to the Cauchy distribution with parameter $N(k+1)$. The first moment does not exist but some fractional moments do, and in particular we have, for $0 \leqslant s<1$,

$$
\begin{aligned}
\mathbb{E}\left[\left|a_{N, k}\right|^{s}\right] & =\frac{N(k+1)}{\pi} \int_{-\infty}^{\infty} \frac{|x|^{s}}{x^{2}+N^{2}(k+1)^{2}} d x \\
& =\frac{1}{\pi} N^{s}(k+1)^{s} \Gamma\left(\frac{1}{2}+\frac{s}{2}\right) \Gamma\left(\frac{1}{2}-\frac{s}{2}\right) .
\end{aligned}
$$

Moreover,

$$
\mathbb{E}\left[\log \left|a_{N, k}\right|\right]=\log (N(k+1)) .
$$

Hence, we can apply Proposition 6 and deduce that the zeros of the sequence of random polynomials with coefficients $\left(a_{N, k}\right)_{\substack{N \leqslant 1 \\ 0 \leqslant k \leqslant N}}$ where $a_{N, k}$ are chosen from the Cauchy distribution with parameter $N(k+1)$ cluster uniformly around the unit circle. 


\section{ZEROS OF RANDOM POLYNOMIALS}

Example 2. For each $N$, let $\left(a_{N, k}\right), 0 \leqslant k \leqslant N$, be discrete random variables taking values in $\{ \pm 1, \ldots, \pm N\}$, not necessarily having the same distribution. Then for any positive function $\alpha_{N} \leqslant N$ such that $\alpha_{N} / \log N \rightarrow \infty$ we have

$$
\begin{aligned}
& \lim _{N \rightarrow \infty} \frac{1}{N} \nu_{N}\left(\frac{\alpha_{N}}{N}\right)=1, \quad \text { almost surely, } \\
& \frac{1}{N} \nu_{N}(\theta, \phi) \rightarrow \frac{\phi-\theta}{2 \pi}, \quad \text { almost surely. }
\end{aligned}
$$

This follows from Theorem 5 , since for any choice of the $a_{N, k}$ we have the deterministic bound

$$
\begin{aligned}
L_{N} & =\log \left(\sum_{k=0}^{N}\left|a_{N, k}\right|\right)-\frac{1}{2} \log \left|a_{N, 0}\right|-\frac{1}{2} \log \left|a_{N, N}\right| \\
& \leqslant \log ((N+1) N)<2 \log (N+1)
\end{aligned}
$$

with this choice of $\alpha_{N}, L_{N}=o\left(\alpha_{N}\right)$ almost surely.

As a special case, we have the well-known random polynomials $\sum_{k=0}^{N} \mu_{k} Z^{k}$, where $\mu_{k}= \pm 1$, with probabilities $p$ and $(1-p)$. Moreover, we have from the Markov inequality, the following rate for the convergence in probability: There exists a constant $C$ such that

$$
\begin{aligned}
\mathbb{P}\left\{\left(1-\frac{1}{N} \nu_{N}\left(\frac{\alpha_{N}}{N}\right)\right)>\varepsilon\right\} \leqslant \frac{1}{\varepsilon} \frac{C \log N}{\alpha_{N}} \\
\mathbb{P}\left\{\left|\frac{1}{N} \nu_{N}(\theta, \phi)-\frac{\phi-\theta}{2 \pi}\right|>\varepsilon\right\} \leqslant \frac{1}{\varepsilon^{2}} \frac{C \log N}{N}
\end{aligned}
$$

for any fixed $\varepsilon>0$.

\subsection{Classical random polynomials}

Let us now consider the special, but important case of the classical random polynomials as mentioned in $\S 1$, that is

$$
P_{N}(Z)=\sum_{k=0}^{N} a_{k} Z^{k} .
$$

These polynomials have been extensively studied (see, for example, [BS86] or [Far98] for a complete account).

The results of the previous section take a simpler form in the special case of random polynomials of the form (15). The conditions (10) and (11) become

$$
\mathbb{P}\left\{a_{N}=0\right\}=0, \quad \text { for all } N \geqslant 0 .
$$

In this more special case, we can deal more easily with almost sure convergence, which in our framework is the strongest convergence. We proceed to give some simple-to-check sufficient conditions on the moments of $a_{k}$ to ensure that Theorem 5 holds.

Theorem 7. Let $\left(a_{k}\right)_{k \geqslant 0}$ be a sequence of complex random variables. Assume that the following hold.

- There exists some $s>0$ such that if $\lambda_{k}:=\mathbb{E}\left[\left|a_{k}\right|^{s}\right]$, then $\lambda_{k}<\infty$ for all $k$, and

$$
\limsup _{k \rightarrow \infty}\left(\lambda_{k}\right)^{1 / k}=1 \text {. }
$$

- There exists some $t>0$ such that for all $k$,

$$
\xi_{k}:=\mathbb{E}\left[\frac{1}{\left|a_{k}\right|^{t}}\right]<\infty
$$




\section{P. Hughes And A. Nikeghbali}

and

$$
\limsup _{k \rightarrow \infty}\left(\xi_{k}\right)^{1 / k}=1
$$

Then there exists a deterministic positive sequence $\left(\alpha_{N}\right)$ subject to $0<\alpha_{N} \leqslant N$ for all $N$ and $\alpha_{N}=o(N)$ as $N \rightarrow \infty$, such that

$$
\lim _{N \rightarrow \infty} \frac{1}{N} \nu_{N}\left(\frac{\alpha_{N}}{N}\right)=1, \quad \text { almost surely }
$$

and

$$
\lim _{N \rightarrow \infty} \frac{1}{N} \nu_{N}(\theta, \phi)=\frac{\phi-\theta}{2 \pi}, \quad \text { almost surely. }
$$

In fact, the convergence also holds in the pth mean for every positive $p$.

The proof of this theorem requires the following simple lemma.

LEMmA 8. If $\left(\lambda_{k}\right)$ is a sequence of real non-negative numbers such that

$$
\limsup _{k \rightarrow \infty} \lambda_{k}^{1 / k} \leqslant 1
$$

then there exists a sequence $\left(\varepsilon_{N}\right)$ of real positive numbers tending to zero such that

$$
\sum_{k=0}^{N} \lambda_{k} \leqslant \exp \left(N \varepsilon_{N}\right)
$$

for all $N$.

Proof. Let $\epsilon>0$ be arbitrarily small. Since $\lim _{\sup _{k \rightarrow \infty}} \lambda_{k}^{1 / k} \leqslant 1$ there exists a constant $C=C(\epsilon)$ such that $\lambda_{k} \leqslant C(1+\epsilon)^{k}$. Therefore,

$$
\begin{aligned}
\sum_{k=0}^{N} \lambda_{k} & \leqslant C \frac{(1+\epsilon)^{N+1}-1}{\epsilon} \\
& \leqslant \exp \left(N\left(\epsilon+\frac{\log C-\log \epsilon+\epsilon}{N}\right)\right),
\end{aligned}
$$

where we have used the bound $\log (1+\epsilon) \leqslant \epsilon$ for $\epsilon>0$. Hence, there exists an $N_{0}=N_{0}(\epsilon)$ such that for all $N>N_{0}$

$$
\sum_{k=0}^{N} \lambda_{k} \leqslant \exp (2 N \epsilon),
$$

and since $\epsilon$ is arbitrary, this proves the lemma.

Proof of Theorem \%. Note that (16) implies $\mathbb{P}\left\{\left|a_{k}\right|=0\right\}=0$. Therefore, from Theorem 5 it is sufficient to prove that there exists a deterministic sequence $\alpha_{N}=o(N)$ such that $\left(1 / \alpha_{N}\right) L_{N} \rightarrow 0$ almost surely, which by the Borel-Cantelli lemma would follow from showing that for any $\epsilon>0$,

$$
\sum_{N=1}^{\infty} \mathbb{P}\left\{\left|\frac{1}{\alpha_{N}} L_{N}\right| \geqslant \epsilon\right\}<\infty .
$$




\section{ZEROS OF RANDOM POLYNOMIALS}

First, from the concavity of log, we note that $L_{N}$ is positive since

$$
\begin{aligned}
L_{N} & :=\log \left(\sum_{k=0}^{N}\left|a_{k}\right|\right)-\frac{1}{2} \log \left|a_{0}\right|-\frac{1}{2} \log \left|a_{N}\right| \\
& \geqslant \log \left(\left|a_{0}\right|+\left|a_{N}\right|\right)-\frac{1}{2} \log \left|a_{0}\right|-\frac{1}{2} \log \left|a_{N}\right| \\
& \geqslant \log 2 .
\end{aligned}
$$

Now, using the fact that

$$
\left\{\frac{1}{\alpha_{N}} L_{N} \geqslant \epsilon\right\} \subseteq\left\{\frac{1}{\alpha_{N}} \log \left(\sum_{k=0}^{N}\left|a_{k}\right|\right) \geqslant \epsilon / 3\right\} \cup\left\{\frac{-\log \left|a_{0}\right|}{2 \alpha_{N}} \geqslant \epsilon / 3\right\} \cup\left\{\frac{-\log \left|a_{N}\right|}{2 \alpha_{N}} \geqslant \epsilon / 3\right\}
$$

we have

$$
\begin{aligned}
\mathbb{P}\left\{\left|\frac{1}{\alpha_{N}} L_{N}\right| \geqslant \epsilon\right\} & =\mathbb{P}\left\{\frac{1}{\alpha_{N}} L_{N} \geqslant \epsilon\right\} \\
& \leqslant \mathbb{P}\left\{\frac{1}{\alpha_{N}} \log \left(\sum_{k=0}^{N}\left|a_{k}\right|\right) \geqslant \epsilon / 3\right\}+\mathbb{P}\left\{\frac{-\log \left|a_{0}\right|}{2 \alpha_{N}} \geqslant \epsilon / 3\right\}+\mathbb{P}\left\{\frac{-\log \left|a_{N}\right|}{2 \alpha_{N}} \geqslant \epsilon / 3\right\} .
\end{aligned}
$$

To calculate the first sum we wish to bound

$$
\mathbb{P}\left\{\sum_{k=0}^{N}\left|a_{k}\right| \geqslant e^{\epsilon \alpha_{N} / 3}\right\}
$$

For this event to happen at least one of the $\left|a_{k}\right|$ must be larger than $e^{\epsilon \alpha_{N} / 3} /(N+1)$. Therefore,

$$
\begin{aligned}
\mathbb{P}\left\{\sum_{k=0}^{N}\left|a_{k}\right| \geqslant e^{\epsilon \alpha_{N} / 3}\right\} & \leqslant \mathbb{P}\left\{\bigcup_{k=0}^{N}\left\{\left|a_{k}\right| \geqslant \frac{1}{N+1} e^{\epsilon \alpha_{N} / 3}\right\}\right\} \\
& \leqslant \sum_{k=0}^{N} \mathbb{P}\left\{\left|a_{k}\right| \geqslant \frac{1}{N+1} e^{\epsilon \alpha_{N} / 3}\right\} .
\end{aligned}
$$

By Tchebychev's inequality we have

$$
\mathbb{P}\left\{\left|a_{k}\right| \geqslant \frac{1}{N+1} e^{\epsilon \alpha_{N} / 3}\right\} \leqslant \frac{\mathbb{E}\left[\left|a_{k}\right|^{s}\right]}{\left(1 /(N+1) e^{\epsilon \alpha_{N} / 3}\right)^{s}}=\lambda_{k}(N+1)^{s} \exp \left(-s \epsilon \alpha_{N} / 3\right) .
$$

Summing this over $k$ from 0 to $N$, and using Lemma 8 , we see that there exists a sequence of positive numbers $\left(\varepsilon_{N}\right)$ which tends to zero such that

$$
\mathbb{P}\left\{\sum_{k=0}^{N}\left|a_{k}\right| \geqslant e^{\epsilon \alpha_{N} / 3}\right\} \leqslant \exp \left(s \log (N+1)-s \epsilon \alpha_{N} / 3+N \varepsilon_{N}\right) .
$$

Hence if, for $N \geqslant N_{0}$ for some $N_{0}$ sufficiently large, $\alpha_{N}$ is chosen so that

$$
\alpha_{N} \geqslant \frac{3 N \varepsilon_{N}}{s \epsilon}+\left(\frac{3}{\epsilon}+\frac{6}{s \epsilon}\right) \log (N+1)
$$

and subject to the extra conditions $0<\alpha_{N} \leqslant N$ with $\alpha_{N}=o(N)$, then we see that

$$
\sum_{N=N_{0}}^{\infty} \mathbb{P}\left\{\sum_{k=0}^{N}\left|a_{k}\right| \geqslant e^{\epsilon \alpha_{N} / 3}\right\} \leqslant \sum_{N=1}^{\infty} \frac{1}{(N+1)^{2}}<\infty
$$


and, hence,

$$
\sum_{N=1}^{\infty} \mathbb{P}\left\{\sum_{k=0}^{N}\left|a_{k}\right| \geqslant e^{\epsilon \alpha_{N} / 3}\right\}<\infty .
$$

We now deal with the remaining two terms in (17). If $\alpha_{N} / \log N \rightarrow \infty$, then by Tchebychev's inequality and the assumption that $\mathbb{E}\left[\left|a_{0}\right|^{-t}\right]<\infty$, we have

$$
\sum_{N=1}^{\infty} \mathbb{P}\left\{\frac{-\log \left|a_{0}\right|}{2 \alpha_{N}} \geqslant \epsilon / 3\right\} \leqslant \sum_{N=1}^{\infty} \frac{\mathbb{E}\left[\left|a_{0}\right|^{-t}\right]}{\exp \left(2 t \epsilon \alpha_{N} / 3\right)}<\infty .
$$

Finally note that $\lim \sup _{N \rightarrow \infty} \xi_{N}^{1 / N} \leqslant 1$ implies there exists a positive sequence $\left(\varepsilon_{N}^{\prime}\right)$ tending to zero such that $\xi_{N} \leqslant \exp \left(N \varepsilon_{N}^{\prime}\right)$ for all $N$. Hence, by (16) and Tchebychev's inequality,

$$
\begin{aligned}
\sum_{N=1}^{\infty} \mathbb{P}\left\{\frac{-\log \left|a_{N}\right|}{2 \alpha_{N}} \geqslant \epsilon / 3\right\} & \leqslant \sum_{N=1}^{\infty} \mathbb{E}\left[\frac{1}{\left|a_{N}\right|^{t}}\right] \exp \left(-2 t \epsilon \alpha_{N} / 3\right) \\
& =\sum_{N=1}^{\infty} \xi_{N} \exp \left(-2 t \epsilon \alpha_{N} / 3\right) \\
& \leqslant \sum_{N=1}^{\infty} \exp \left(N \varepsilon_{N}^{\prime}-2 t \epsilon \alpha_{N} / 3\right)
\end{aligned}
$$

and this sum is finite if

for sufficiently large $N$.

$$
\alpha_{N} \geqslant \frac{3 N \varepsilon_{N}^{\prime}}{2 t \epsilon}+\frac{3}{t \epsilon} \log N
$$

Combining the previous two equations and (18) with (17) we conclude that if $\left(\alpha_{N}\right)$ is a deterministic sequence satisfying $0<\alpha_{N} \leqslant N$ for all $N, \alpha_{N}=o(N)$ and

$$
\frac{\alpha_{N}}{\log N+N \varepsilon_{N}+N \varepsilon_{N}^{\prime}} \rightarrow \infty
$$

(and such a sequence exists since both $\varepsilon_{N}$ and $\varepsilon_{N}^{\prime}$ tend to zero), then for any $\epsilon>0$,

$$
\sum_{N=1}^{\infty} \mathbb{P}\left\{\left|\frac{1}{\alpha_{N}} L_{N}\right| \geqslant \epsilon\right\}<\infty
$$

which by the Borel-Cantelli lemma shows $L_{N} / \alpha_{N} \rightarrow 0$ almost surely, and the result now follows from Theorem 5 .

From Theorem 7 we deduce the following corollary.

COROLlary 9. Let $\left(a_{k}\right)_{k \geqslant 0}$ be a sequence of (possibly dependent) complex random variables such that the moduli $\left|a_{k}\right|$ have densities which are uniformly bounded in a neighbourhood of the origin. Assume that there exists some $s \in(0,1]$ such that if $\lambda_{k}:=\mathbb{E}\left[\left|a_{k}\right|^{s}\right]$ then $\lambda_{k}<\infty$ for all $k$, and

$$
\limsup _{k \rightarrow \infty}\left(\lambda_{k}\right)^{1 / k}=1 .
$$

Then almost surely the zeros of the classical random polynomial

$$
P(Z)=\sum_{k=0}^{N} a_{k} Z^{k}
$$

cluster uniformly around the unit circle. 


\section{ZEROS OF RANDOM POLYNOMIALS}

Proof. It suffices to note that in this special case, $\sup _{N} \xi_{N} \leqslant C$ for some positive constant $C$, and so the conclusions of Theorem 7 follow. These imply uniform clustering of the zeros (and even give an estimate on the rate of clustering).

Example. Let $P_{N}(Z)=\sum_{k=0}^{N} a_{k} Z^{k}$, with $a_{k}$ being distributed on $\mathbb{R}_{+}$with Cauchy distribution with parameter $k^{-\sigma}, \sigma>0$. This distribution has density

$$
\frac{2}{\pi k^{\sigma}} \frac{1}{x^{2}+k^{-2 \sigma}}
$$

on the positive real line. The conditions of Theorem 7 are satisfied since

$$
\lambda_{k}:=\mathbb{E}\left[a_{k}^{1 / 2}\right] \leqslant \frac{C}{k^{\sigma}} \quad \text { and } \quad \xi_{N}:=\mathbb{E}\left[\frac{1}{a_{N}^{1 / 2}}\right] \leqslant C k^{\sigma} .
$$

Therefore, if $\alpha_{N}=o(N)$ is such that $\alpha_{N} / \log N \rightarrow \infty$, then

$$
\lim _{N \rightarrow \infty} \frac{1}{N} \nu_{N}\left(\frac{\alpha_{N}}{N}\right)=1, \quad \text { almost surely }
$$

and

$$
\lim _{N \rightarrow \infty} \frac{1}{N} \nu_{N}(\theta, \phi)=\frac{\phi-\theta}{2 \pi}, \quad \text { almost surely. }
$$

Again, the convergence also holds in the $p$ th mean for every positive $p$.

\section{ACKNowledGements}

This work was partly carried out at the Random Matrix Approaches in Number Theory programme held at the Isaac Newton Institute, where the first author was supported by EPSRC grant N09176, and partly at the American Institute of Mathematics. Both authors were partially supported by a NSF Focussed Research Group grant 0244660. We wish to thank David Farmer, Steve Gonek, Francesco Mezzadri, Andrew Wade, and Marc Yor for useful conversations. We also wish to thank the referee for careful reading of this paper and finding a mistake in an earlier draft.

\section{REFERENCES}

Arn66 L. Arnold, Über die Nullstellenverteibung zufälliger polynome, Math. Z. 92 (1966), 12-18.

BS86 A. T. Bharucha-Reid and M. Sambandham, Random polynomials, in Probability and Mathematical Statistics (Academic Press, Orlando, FL, 1986).

Bil97 Y. Bilu, Limit distribution of small points on algebraic tori, Duke Math. J. 89 (1997), 465-476.

BBL96 E. Bogomolny, O. Bohigas and P. Leboeuf, Quantum chaotic dynamics and random polynomials, J. Statist. Phys. 85 (1996), 639-679.

EK95 A. Edelman and E. Kostlan, How many zeros of a random polynomial are real?, Bull. Amer. Math. Soc. 32 (1995), 1-37.

ET50 P. Erdős and P. Turán, On the distribution of roots of polynomials, Ann. of Math. (2) 51 (1950), $105-119$.

Far98 K. Farahmand, Topics in random polynomials, Pitman Research Notes in Mathematics Series, vol. 393 (Longman, Harlow, 1998).

Lan99 S. Lang, Complex analysis, fourth edition, Graduate Texts in Mathematics, vol. 103 (Springer, New York, 1999).

SH02 E. Shmerling and K. J. Hochberg, Asymptotic behavior of roots of random polynomial equations, Proc. Amer. Math. Soc. 130 (2002), 2761-2770.

SS62 D. I. Šparo and M. G. Šur, On the distribution of roots of random polynomials, Vestnik Moskov. Univ. Ser. I Mat. Mekh. 1962 (1962), 40-43. 


\section{ZEROS OF RANDOM POLYNOMIALS}

C. P. Hughes ch540@york.ac.uk

Department of Mathematics, University of York, York, YO10 5DD, UK

A. Nikeghbali ashkan.nikeghbali@math.unizh.ch

Institut für Mathematik, Universität Zürich, Winterthurerstrasse 190, CH-8057 Zürich, Switzerland 